\title{
Terrestrial Plants Molluscicidal to Lymnaeid Hosts of Fascioliasis Hepatica in Puerto Rico ${ }^{1}$
}

\author{
Freddy R. Medina and Roy Woodbury ${ }^{2}$
}

\begin{abstract}
Molluscicidal assay of 200 Puerto Rican plants revealed 30 to be active against Lymnaea cubensis and $L$. columella under laboratory screening. Among these 30,16 were notably toxic against lymnaeids, killing all snails in the range of 25 to $200 \mathrm{p} / \mathrm{m}$. Of plant parts tested, including seeds, roots, fruits and leaves, the latter two proved most uniformly toxic. Only in the case of Solanum nodifiorum were all parts toxic. This paper discusses how molluscicidal plants may be beneficially used in the field to control snail-borne diseases, and what qualities an ideal molluscicidal plant should have.
\end{abstract}

\section{INTRODUCTION}

Extensive literature attests to the great efforts made to control fascioliasis since its European discovery in 1379. It continues as a major agricultural problem adversely affecting ovine and bovine productivity. Man is an occasional victim of Fasciola hepatica in some 20 countries; it is a serious public health problem in Perú (1).

Factors in the control of fascioliasis are health education, and hygiene, improved farm management, developments in chemotherapy, vaccination, and destruction of snail intermediate hosts. Our interest is the possible use of molluscicidal plants for controlling the Puerto Rican snail vectors Lymnaea columella and L. cubensis.

Although much progress has been made in chemical poisoning of snail vectors of the major human trematodiases, such as in schistosomiasis, methods provided do not fit the requirements of fascioliasis control. Among several problems, there is the possibility of harming agricultural herbs or crops through dispersion of toxic chemicals. Since the snail vectors are commonly amphibious, their pattern of dispersal is extensive and poorly defined, making it difficult to expose all to molluscicides. Alternatively, we seek new solutions to an ancient problem under the aegis of biological control of the snail vectors. Naturalistic methods for killing snails are varied $(2,7,13)$ and provide many research leads. In Puerto Rico, a number of studies indicate promise of success. Marisa, a demonstrated predator of other fresh water snails, apparently destroyed colonies of Lymnaea columella, as well as Biomphalaria glabrata (16). The same was indicated in a laboratory study (4) against $L$. caillaudi

\footnotetext{
${ }^{1}$ Manuscript submitted to Editorial Board July 18, 1978.

${ }^{2}$ Puerto Rico Junior College, Río Piedras, P.R., and Department of Biology, University of Puerto Rico, Río Piedras, P.R. Drs. Lawrence S. Ritchie and Frederick F. Ferguson assisted in the preparation of the manuscript.
} 
(the African vector of fascioliasis gigantica). Marisa is used routinely by the Puerto Rico Department of Health for sppressing populations of $B$. glabrata in control of schistosomiasis $(15,17)$. Berg and co-workers have clearly shown that the specific predation of freshwater snails by hundreds of species of sciomyzid marsh-fly larvae is worthly of definitive field trials in trematode control (2). Studies are in progress using the native Sepedon caerulia, for the purpose of controlling our lymnaeid vectors of fascioliasis (5). The aquatic annelid Chaetogaster limnaei has been shown to destroy snails and their eggs, cercaria, and miracidia of Fasciola hepatica (1). Preliminary tests of the ability of this predatory annelid to control lymnaeids are in progress. ${ }^{3}$ Paralleling the above efforts, we sought yet another means of bringing abating pressure to bear upon Lymnaea utilizing toxic materials inherent in local land plants.

The literature in this little known field has been reviewed by Bond (3). It reflects empirical studies of many plant materials, for example, the saponins (14). The hope that an effective plant molluscicide derived from Endod (Phytolocca dodecandra) could be employed to control schistosomiasis in Ethiopia has received well deserved notice (9). In that program Endod powder is mass produced for ordinary application in endemic bodies of water. Our ultimate intention is to search out those plants that have useful molluscicidal properties against Lymnaea and to grow the toxic vegetation in or at the margins of snail habitats. Leaf or fruit might provide toxic transport in some cases, while root exudates might suffice in others. This study was a survey to find molluscicidal plants that may be used in this manner as biological control against the snail vectors of fascioliasis hepatica.

\section{MATERIALS AND METHODS}

Two hundred plants listed in tables 1 and 2 were collected in the northern part of Puerto Rico; two test plants were from the Dominican Republic. The criteria used for the selection of the plants were 1) genera of plants known to be toxic against snails; 2) plants known to have medicinal effects in mammals; 3) plants toxic for mammals; and 4) randomly selected plants from lymnaeid habitats.

Lymnaea cubensis and $L$. columella were the two snail species used in this work. These snails were reared under standard laboratory conditions. L. cubensis was cultured in a mud tray nurtured on an algal overgrowth. $L$. columella was reared in aquaria with water two in deep. A supplementary food formula consisting of alfalfa powder, wheatgerm, Dog Chow, powdered milk (4:2:2:1) and calcium chloride (3.8\% of total volume) was also provided for both species.

${ }^{3}$ P. Bendezú, personal communication, 1974. 
TABLE 1,-Molluscicidal plants-lethal concentrations (100\%)

\begin{tabular}{|c|c|c|c|c|}
\hline Family name & Genus and species & Common names & Toxic parts $^{1}$ & $\mathrm{P} / \mathrm{m}$ \\
\hline Alpinaceae & Hedychium coronarium $^{2}$ & Nardo, Jazmín del río & Seeds & 25 \\
\hline Apocynaceae & Allamanda cathartica ${ }^{2}$ & Canario & Leaves & 1000 \\
\hline Asclepidaceae & Asclepias curassavica & Algodoncillo & Roots & 100 \\
\hline Burseraceae & Bursera simaruba & Almácigo & Seeds & 1000 \\
\hline Basidiomicetae & Unidentified Basidiomycete & Unknown & All Parts & 1000 \\
\hline Casuarinaceae & Casuarina equisetifolia & Pino & Fruits and Leaves & 100 and 1000 \\
\hline Euphorbiaceae & Jatropha gossypifolium & Tuatúa & Leaves & 1000 \\
\hline \multirow[t]{3}{*}{ Fabeceae } & Andira inermis & Moca & Roots & 1000 \\
\hline & Cajanus cajan & Gandul & Roots and Leaves & 1000 \\
\hline & Indigofera suffruticosa & Añil & Seeds & 100 \\
\hline \multirow[t]{2}{*}{ Mimosaceae } & Inga vera & Guava & Roots and bulbs & 1000 \\
\hline & Samanea saman ${ }^{2}$ & Samán & Leaves & 1000 \\
\hline \multirow[t]{8}{*}{ Solanaceae } & Capsicum frutescens & Ají & Leaves and Fruits & 100 \\
\hline & Cestrum diurnum & Dama del día & Leaves and Mature Fruits & 1000 \\
\hline & Cestrum laurifolium & Galán del monte & Leaves and Mature Fruits & 100 \\
\hline & Cestrum macrophyllum ${ }^{4}$ & Galán del monte & Leaves & 100 \\
\hline & Lycopersicon esculentum & Tomate & Leaves & 1000 \\
\hline & Solanum nodiflorum & Yerba mora & All Parts & 100 \\
\hline & Solanum torvum & Berenjena cimarrona & Leaves & 1000 \\
\hline & Solanum mammosum & Berenjena de cucaracha & Mature Fruits (Pulp) & 100 \\
\hline Verbenaceae & Clerodendron fragans ${ }^{2}$ & Flor de muerto & Leaves & 1000 \\
\hline
\end{tabular}

' Roots, leaves and fruits of all plants were tested separately with the exception of those marked with ${ }^{2}$ and ${ }^{4}$ footnotes.

${ }^{2}$ Roots not tested.

${ }^{3}$ Collected in Dominican Republic at 3,000 feet above sea level and in Puerto Rico at El Verde.

${ }^{4}$ Only leaves tested. 
TABLE 2.-Non-molluscicidal plants

\begin{tabular}{|c|c|c|}
\hline Family name & Genus and species & Common name \\
\hline Acanthaceae & $\begin{array}{l}\text { Crossandra infundibuliformis } \\
\text { Justicia verticillata } \\
\text { Rusellia equisetiformis } \\
\text { Stethoma pectoralis }\end{array}$ & $\begin{array}{l}\text { Doña Juana } \\
\text { Unknown } \\
\text { Lluvia de coral } \\
\text { Curia }\end{array}$ \\
\hline Alpinaceae & $\begin{array}{l}\text { Alpinia purpurata } \\
\text { Alpinia speciosa }\end{array}$ & $\begin{array}{l}\text { Purple Ginger } \\
\text { Shell flower }\end{array}$ \\
\hline Alismaceae & Sagittaria lancifolia & Saeta de agua \\
\hline Amaranthaceae & $\begin{array}{l}\text { Amaranthus paniculata } \\
\text { Achyranthes aspera } \\
\text { Petiveria alliacea }\end{array}$ & $\begin{array}{l}\text { Unknown } \\
\text { Unknown } \\
\text { Anamú }\end{array}$ \\
\hline Amarilidaceae & $\begin{array}{l}\text { Agave angustifolia } \\
\text { Agave furcryoides }\end{array}$ & $\begin{array}{l}\text { Agave } \\
\text { Henequén }\end{array}$ \\
\hline Amilaceae & Centella asiatica & Yerba de chavos \\
\hline Anacardiaceae & Mangifera indica & Mango \\
\hline Annonaceae & $\begin{array}{l}\text { Annona glabra } \\
\text { Annona muricata }\end{array}$ & $\begin{array}{l}\text { Corazón cimarrón } \\
\text { Guanábana }\end{array}$ \\
\hline Apocinaceae & Nerium oleander & Alelí \\
\hline Araceae & $\begin{array}{l}\text { Dieffenbachia seguine } \\
\text { Philondendron dubius } \\
\text { Syngonium podophylum }\end{array}$ & $\begin{array}{l}\text { Rábano } \\
\text { Yautía type } \\
\text { Unknown }\end{array}$ \\
\hline Araliaceae & Aralia balfouriana & Arelia \\
\hline Asclepidaceae & Cryptostegia grandiflora & Bejuco de goma \\
\hline Balsaminaceae & $\begin{array}{l}\text { Impatiens balsamina } \\
\text { Impatiens sultani }\end{array}$ & $\begin{array}{l}\text { Espuela de galán } \\
\text { Miramelinda }\end{array}$ \\
\hline Begoniaceae & Begonia sp. & Begonia \\
\hline Bignoniaceae & Crescentia cujete & Higüero \\
\hline Bixiaceae & Bixia orellana & Achiote \\
\hline Boraginaceae & Heliotropium indicum & Cotorrera \\
\hline Boraginaceae & Tournefortia hirsutissima & Nigua \\
\hline Bromeliaceae & $\begin{array}{l}\text { Bromelia pyramidalis } \\
\text { Bromelia sp. }\end{array}$ & $\begin{array}{l}\text { Bromelia } \\
\text { Bromelia }\end{array}$ \\
\hline Cactaceae & $\begin{array}{l}\text { Hylocereus trigonus } \\
\text { Cactus sp. }\end{array}$ & $\begin{array}{l}\text { Pitahaya } \\
\text { Ornamental cactus }\end{array}$ \\
\hline Cambretaceae & Bucida buceras & Ucar \\
\hline Cannaceae & Canna sp. & Maraca \\
\hline Cesalpinaceae & $\begin{array}{l}\text { Biancaea sepiaria } \\
\text { Cassia occidentalis } \\
\text { Cassia mimosioides } \\
\text { Cassia siamea } \\
\text { Cassia tora } \\
\text { Tamarindus indicus }\end{array}$ & $\begin{array}{l}\text { Zarza } \\
\text { Hedionda } \\
\text { Morivivi falso } \\
\text { Casia } \\
\text { Hedionda } \\
\text { Tamarindo }\end{array}$ \\
\hline Characeae & Chara sp. & Chara \\
\hline Commelinaceae & $\begin{array}{l}\text { Commelina virginiana } \\
\text { Rhoeo discolor }\end{array}$ & $\begin{array}{l}\text { Cohitre } \\
\text { Sangria }\end{array}$ \\
\hline Compositae & $\begin{array}{l}\text { Bidens pilosa } \\
\text { Calendula erecta } \\
\text { Mikania cordifolia } \\
\text { Mikania fragilis } \\
\text { Parthenium hysterophorum } \\
\text { Pluchea odorata } \\
\text { Pseudoelephantopus spicatus } \\
\text { Wedelia trilobata }\end{array}$ & $\begin{array}{l}\text { Margarita silvestre } \\
\text { Caléndula } \\
\text { Guaco } \\
\text { Unknown } \\
\text { Ajenjo cimarrón } \\
\text { Salvia } \\
\text { Lengua de vaca } \\
\text { Manzanilla de playa }\end{array}$ \\
\hline
\end{tabular}


TABle 2.-Continued

\begin{tabular}{|c|c|c|}
\hline Family name & Genus and species & Common name \\
\hline \multirow[t]{3}{*}{ Convolvulaceae } & Ipomoea rosea & Bejuco de puerco \\
\hline & Ipomoea tiliacea & Bejuco de puerco \\
\hline & Ipomoea triloba & Bejuco de puerco, bejuquillo \\
\hline \multirow[t]{2}{*}{ Crasulaceae } & Bryophyllum pinnatum & Bruja \\
\hline & Crassula sp. & Unknown \\
\hline \multirow[t]{2}{*}{ Cucurbitaceae } & Cayaponia americana & Bejuco de torero \\
\hline & Mormodica charantia & Cundeamor \\
\hline \multirow[t]{4}{*}{ Cyperaceae } & Cupania americana & Guará blanca \\
\hline & Cyperus alternifolius & Paragüita \\
\hline & Cyperus giganteus & Junco de ciénaga \\
\hline & Eleocharis interstincta & Junco de aparejos \\
\hline \multirow[t]{2}{*}{ Dioscoreaceae } & Dioscorea aculeata & Name tongo \\
\hline & Rajania cordata & Guayaro \\
\hline \multirow[t]{2}{*}{ Eretiaceae } & Bourreria succulenta & Palo de vaca \\
\hline & Cordia corymbosa & Capa \\
\hline \multirow[t]{11}{*}{ Euphorbiaceae } & Alcalypha hispida & Hot pocker \\
\hline & Hura crepitans & Molinillo \\
\hline & Jatropha curca & Pin̄ón, tártago \\
\hline & Jatropha grandifolia & Tobillo \\
\hline & Manihot esculenta & Yuca \\
\hline & Phyllanthus acidus & Grosella \\
\hline & Phyllanthus niruri & Viernes Santo \\
\hline & Phyllanthus nobilis & Avispillo \\
\hline & Poinsettia pulcherrima & Flor de pascua \\
\hline & Ricinus communis & Higuereta \\
\hline & Sapium laurocerasus & Hinchahuevos \\
\hline \multirow[t]{14}{*}{ Fabaceae } & Aeschynomene americana & Moriviví bobo \\
\hline & Centrosema pubescens & Conchita \\
\hline & Crotalaria retusa & Matraca \\
\hline & Crotalaria striata & Matraca \\
\hline & Dalbergia monetaria & Bejuco de chavos \\
\hline & Desmodium canum & Zarzabacoa \\
\hline & Desmodium sp. & Trébol \\
\hline & Erythrina coralmandelianum & Machete \\
\hline & Phaseolus vulagaris & Habichuela \\
\hline & Gliricidia sepium & Mata ratón \\
\hline & Mucuna sloanei & Mato \\
\hline & Ormosia krugii & Palo de matos \\
\hline & Pueraria hirsuta & Kudzú \\
\hline & Tephrosia toxicarcia & Barbasco de raiz \\
\hline Gesneriaceae & Gesneria albiflora & Unknown \\
\hline \multirow[t]{6}{*}{ Gramineae } & Andropogon nardus & Limoncillo \\
\hline & Cynodon dactylon & Bermuda \\
\hline & Eriochloa polystachya & Malojilla \\
\hline & Gynerium sagittifolia & Caña india \\
\hline & Panicum muticum & Malojillo \\
\hline & Panicum maximun & $\begin{array}{l}\text { Yerba de guinea, gramalote, } \\
\text { yerba Borinquen }\end{array}$ \\
\hline Guttifereae & Calophyllum calaba & María \\
\hline
\end{tabular}


TABLE 2.-Continued

\begin{tabular}{|c|c|c|}
\hline Family name & Genus and species & Common name \\
\hline Hippocrateaceae & Hippocratea volubilis & Bejuco prieto \\
\hline Iridaceae & Tigrida pavonia & Bejuco violeta \\
\hline \multirow[t]{3}{*}{ Lamiaceae } & Ocimum basilicum & Albahaca \\
\hline & Coleus blumei & Cóleo \\
\hline & Ocimum officinalis & Albahaca blanca \\
\hline Lauraceae & Persea americana & Aguacate \\
\hline \multirow[t]{4}{*}{ Liliaceae } & Aloe barbadensis & Sábila \\
\hline & Aloe ferox & Závila de jardín \\
\hline & Yucca aloifolia & Aguja de Adán \\
\hline & Sanseveria guineensis & Lengua de vaca \\
\hline Lythraceae & Lagerstroemia indica & Astromelia \\
\hline Lobeliaceae & Lobelia longiflora & Tibey \\
\hline Lycopodiaceae & Lycopodium cernuum & Azufre vegetal \\
\hline \multirow[t]{2}{*}{ Malvaceae } & Montezuma speciosissima & Maga \\
\hline & Urena lobata & Cadillo de perro \\
\hline Melastomaceae & Miconia prasina & Camasey \\
\hline Meliaceae & Guarea trichiloides & Guaraguao \\
\hline Menispermaceae & Cissampelos pareira & Bejuco de mona \\
\hline Menyanthaceae & Nymphoides humboldtianum & Trébol de agua \\
\hline Mimosaceae & Samanea saman & Saman \\
\hline Moraceae & Ficus indica & Higo chumbo \\
\hline Musaceae & Heliconia psittocorum & Unknown \\
\hline \multirow[t]{3}{*}{ Myrtaceae } & Pimenta racemosa & Malagueta \\
\hline & Eugenia jambos & Pomarrosa \\
\hline & Psidium guajava & Guayaba \\
\hline \multirow[t]{2}{*}{ Nyctaginaceae } & Bougainvillea glabra & Trinitaria roja \\
\hline & Mirabilis jalapa & Don Diego de noche \\
\hline Onagraceae & Ludwigia leptocarpa & Evening primerose \\
\hline \multirow[t]{3}{*}{ Piperaceae } & Piper aduncum & Higuillo hoja menuda \\
\hline & Piper marginatum & Higuillo oloroso \\
\hline & Piper peltata & Piquiña \\
\hline Plantaginaceae & Plantago major & Llantén \\
\hline Polygalaceae & Polygala paniculata & Orosne \\
\hline \multirow{2}{*}{ Polygonaceae } & Antigonon leptopus & Coralillo \\
\hline & Polygonum punctatum & Yerba de hicotea \\
\hline \multirow[t]{3}{*}{ Polypodiaceae } & Dryopteris dentata & Helecho \\
\hline & Nephrolepis biserrata & Unknown \\
\hline & Polypodium polycarpum & Unknown \\
\hline Portulacaceae & Portulaca oleracea & Verdolaga \\
\hline Rosaceae & Rosa sp. & Rosa \\
\hline \multirow[t]{10}{*}{ Rubiaceae } & Borreria ocymoides & Juana la blanca \\
\hline & Borreria verticillata & Botón blanco \\
\hline & Chiococca alba & Bejuco de berac \\
\hline & Diodia sarmentosa & Unknown \\
\hline & Gonzalagunia hirsuta & Rabo de ratón \\
\hline & Hamelia erecta & Bálsamo \\
\hline & Ixora coccinea & Cruz de Malta \\
\hline & Psychotria brachiata & Palo de cachimbo \\
\hline & Psychotria grandis & Espuela de galán \\
\hline & Randia aculeata & Tintillo \\
\hline
\end{tabular}


TABLE 2.-Continued

\begin{tabular}{lll}
\hline \multicolumn{1}{c}{ Family name } & \multicolumn{1}{c}{ Genus and species } & \multicolumn{1}{c}{ Common name } \\
\hline Rutaceae & Citrus aurantifolia & Limón \\
& Ruta chalapensis & Ruda \\
Sapindaceae & Paullinia pinnata & Bejuco de guajanilla \\
& Cupania americana & Guara blanca \\
& Serjania polyphylla & Bejuco de costillas \\
Solanaceae & Datura suaveolens & Campana de París \\
& Datura sp. (hybrid) & Campana rosada \\
& Physalis angulata & Saca-buche \\
& Solanum ciliatum & Berenjena cimarrona \\
& Solanum melongena & Berenjena \\
Terminaliaceae & Solanum seaforthianum & Falsa belladona \\
Typhaceae & Terminalia catappa & Almendra \\
Urticaceae & Typha domingensis & Enéa \\
& Urera baccifera & Ortiga brava \\
Vitaceae & Urera sp. & Unknown \\
Verbenaceae & Cissus sicyoides & Bejuco de caro \\
& Citharexylum fruticosum & Péndula \\
& Lantana camara & Cuencas de oro \\
& Lippia dulcis & Póleo, Orozús \\
& Petitia domingenis & Capa blanca \\
& Stachytarpheta cayennense & Verbena \\
\hline
\end{tabular}

In this study, fresh unweighed small quantities of live roots, leaves and fruits of each plant were cut and ground with water in an omnimixer. Such samples were diluted to fill a water glass $(325 \mathrm{ml})$ and left refrigerated overnight. Snails were added to these simple plant infusions and were observed after 24 and $48 \mathrm{~h}$. These data were compared with untreated controls (snails kept in the same water without plant tissue). Those plants which exhibited toxicity against the snails were selected for a definitive screening. In screening, the plant materials were oven dried $\left(50^{\circ} \mathrm{C}\right)$ and ground (Thomas Mill Model 3) to a fine powder. Pre-exposure water solutions $(1 \%)$ were prepared prior to the test day and kept refrigerated overnight. The $1 \%$ solution without debris was diluted to 100 , 200,500 and $1,000 \mathrm{p} / \mathrm{m}$. Snails were exposed to plant materials $24 \mathrm{~h}$ and observed after a $24-\mathrm{h}$ recovery period. The criteria of snail death were inactivity, shell discoloration and in case of doubt, foul odor upon crushing. The water used was tap water, declorinated and filtered through charcoal, sand and limestone.

\section{RESULTS}

Among 200 plant species (representing 79 families), 30 were found to have molluscicidal activity against $L$. cubensis and $L$. columella. One or more plant parts of 13 species killed all snails at $100 \mathrm{p} / \mathrm{m}$ or less; three other species were effective at $200 \mathrm{p} / \mathrm{m}$. Hedychium coronariun, or night 
jasmin (seeds), was the most molluscicidal, comparatively four times as active as any other of the plants tested. The chemical stability of its active agent also exceeded that of the other plants. Locally it is a favorite plant because of its evening scent. Two other species of the same family (alpinaceae) were not molluscicidal.

In only one species were all plant parts uniformly molluscicidal at 100 $\mathrm{p} / \mathrm{m}$ : Solanum nodiflorum (Solanaceae). Leaves of other plants active at this level included those of Cestrum diurnum. C. laurifolium, C. macrophyllum, Capsicum frutescens (Solanaceae), Polyscias guilfoylei (Araliaceae), Casuarina equisetifolia (Casuarinaceae), and Cajanus cajan. Similarly, the fruits of C. diurnum, C. laurifolium, Capsicum frutescens, S. mammosum (Solanaceae) and Duggena hirsuta (Rubiaceae); the seed of Indigofera suffruticosa (Fabaceae); and the roots of Asclepias curassavica (Asclepidaceae), and Cajanus cajan (Leguminosae) were active at $100 \mathrm{p} / \mathrm{m}$. Although the Cestrum species were about as toxic as $S$. nodiflorum, their active agents lost toxicity when stored as dry powder.

Leaves and fruits of the plants surveyed were more often molluscicidal against lymnaeids than other plant parts. Whereas all parts of S. nodiflorum were toxic, only leaves and fruits of other species of Solanaceae were active. Some species, such as Codiaeum variegatum, sometimes gave positive and at other times negative results within a series of collections from the same area.

The seed cover of Bursera simaruba inhibited the development of lymnaeid eggs with $1000 \mathrm{p} / \mathrm{m}$, while hatching of the fully developed eggs was retarded with $100 \mathrm{p} / \mathrm{m}$. However, the dried, powdered material lost toxicity under storage.

Analysis of plant families under consideration showed a trend of molluscicidal activity for species of Solanaceae, 8 of 14 being toxic at relatively low concentrations. In contrast, only three of 15 species of Fabaceae, two of 12 Rubiaceae, one of six Verbenaceae and two of 13 Euphorbiaceae were found active. No toxic species were found among 9 Compositae and 6 Graminaceae. Comparisons with other families are unwarranted because too few species were tested, e.g., Phytolaccaceae, for which two species were toxic at $200 \mathrm{p} / \mathrm{m}$, (table 3). Thus far, all plants found to be molluscicidal are dicots.

\section{DISCUSSION}

Biological control of lymnaeids by means of toxic plants may warrant continued study, especially if they can be reared where snail populations occur (12). If so, the cost of mollusciciding would be minimized, including expenditures for synthetic chemicals and dispersion equipment; moreover, ecological disruptions might be reduced (6). The use of locally available molluscicidal plants has been encouraged through the research 
of Lemma (10), Lemma et al. (11), Krochmal \& Lequesne (8) and Bond (3). It has been proposed that molluscicidal plants might be used to enhance other biological control measures, and possibly afford synergistic interrelationships (14).

From this study, Solanum nodiflorum was recognized as a favorable selection for comprehensive, molluscicidal investigations, since all parts of the plants were toxic for at least two lymnaeids (12). If such a plant could thrive in swampy areas, fallen leaves, flowers and fruits, and roots as well, might make the habitat unfavorable for snails. Roots of Asclepias

TABLE 3.-Analysis of molluscicidal activity per family, including non-toxic groups for which four (4) or more species were tested

\begin{tabular}{lccc}
\hline \multicolumn{1}{c}{ Family } & $\begin{array}{c}\text { Number } \\
\text { molluscicidal }\end{array}$ & $\begin{array}{c}\text { Species } \\
\text { total }\end{array}$ & Activity \\
\hline Acanthaceae & 0 & & P/m \\
Alpinaceae & 1 & 4 & 25 \\
Apocinaceae & 1 & 3 & 1000 \\
Araceae & 0 & 2 & - \\
Araliaceae & 1 & 3 & 100 \\
Asclepidaceae & 1 & 2 & 100 \\
Basidiomicetae & 1 & 2 & 1000 \\
Burseraceae & 1 & 1 & 1000 \\
Casuarinaceae & 1 & 1 & 100 \\
Cesalpinaceae & 0 & 1 & - \\
Compositae & 0 & 7 & - \\
Cyperaceae & 0 & 9 & - \\
Euphorbiaceae & 2 & 4 & - \\
Fabaceae & 3 & 13 & 100 and 1000 \\
Graminaceae & 0 & 15 & - \\
Mimosaceae & 2 & 6 & 1000 \\
Onagraceae & 1 & 2 & 1000 \\
Oxalidaceae & 1 & 2 & 1000 \\
Phytolacaceae & 2 & 1 & 200 \\
Rubiaceae & 2 & 2 & 100 and 1000 \\
Solanaceae & 8 & 12 & 100 and 1000 \\
Verbenaceae & 1 & 6 & \\
\hline
\end{tabular}

crussavica, as well as other parts, are molluscicidal and this plant does grow in swampy areas. The family Solanaceae includes a number of molluscicidal members for consideration.

Seeds of Hedychium coronarium were the most molluscicidal plant material tested, and this species is also active against miracidia and cercariae of Schistosoma mansoni (18).

Considerations might be given to toxicities of germinating seeds and seedlings. Furtheremore, it might be appropriate to survey for absence or occurrence of molluscicidal plants in favorable natural habitats of lymnaeids and other trematode vectors, especially $B$. glabrata, and for 
unusual mortalities, as was done by Lemma (10).

Although all but one of the plants found to be molluscicidal have been dicots, the number of families and species involved in this study are too few to generalize. Absence of snails in habitats with dense monocot vegetation has been noted repeatedly, even when snails occur in conjoined foci. However, it may be the density rather than the kinds of plants that preclude habitation.

We believe, that the desirable features of a molluscicidal plant include 1) plant adaptability to wet environment; 2) dispersibility of the plant parts (leaves or fruits) in the habitat; 3 ) release of toxic substances from roots into water or soil; 4) stability of the toxic agent in water; and 5) no toxicity for cattle or sheep, and other organisms in the same habitat.

\section{RESUMEN}

En un ensaye por eliminación selectiva realizado en el laboratorio, se estudiaron las propiedades molusquicidas de 200 plantas de Puerto Rico. El estudio reveló que de las 200,30 atacan a Lymnaea cubensis y $L$. columella. Dieciséis de las 30 fueron muy tóxicas para las limnéideas, pues a concentraciones de 25 a 200 p.p.m. todos los caracoles murieron. De las varias partes de las plantas que se estudiaron-semillas, raíces, frutas y hojas-las últimas dos fueron las más tóxicas. Solamente en el caso de Solanum nodiflorum, todas fueron tóxicas. Se discute cómo estas plantas molusquicidas podrian usarse en el control de enfermedades portadas por caracoles, así como las cualidades que deben tener.

\section{LITERATURE CITED}

1. Bendezú, P., 1970. Algunos aspectos de la epidemiología de la distomatosis hepática y su control biológico en el Valle del Mantaro, IVITA, Lima, Perú. 4to. Bol Extr: 356367.

2. Berg, C. O., 1964. Snail control in trematode diseases: the positive value of sciomyzid larvae, snail-killing diptera, Advan. Parasitol. 2: 259-309.

3. Bond, H., 1974. Argument in favour of the use of natural products, particularly plant products, as molluscicides. Submitted for publication, Wld. Hlth. Org.

4. Demian, E. S. and Lutfy, R. G., 1964. Prospects of the use of Marisa cornuarietis in the biological control of Lymnaea ciallaliaudi in the U. A. R., Proc. Egypt. Acad. Sci. 18: 46-53.

5. Durán-Sands, E. M., 1975. Biología de la relación depredador-presas entre la mosca de ciénaga Sepedon caerulea (Diptera, Sciomyzidae) y los caracoles hospederos intermediarios de Fasciola hepatica en Puerto Rico, Lymnaea cubensis y Lymnaea columella. Thesis, University of Puerto Rico, Rio Piedras, P. R.

6. Ferguson, F. F., 1972. Trends toward biological control of schistosomiasis snails, Proc. Symp. Future of Schistosomiasis Control. New Orleans 85-91.

7. Ferguson, F. F, 1975. Role of biological agents in the control of Schistosome-bearing snails. USPHS-CDC, Atlanta.

8. Krochmal, A. and Lequesne, P. W., 1970. Phytolacca americana: possible source of a molluscicide, U. S. For. Serv. Res. PAP NE 177: 1-8.

9. Lemma, A., 1965. A preliminary report on the molluscicidal property of Endod (Phy. tolacca dodecandra), Ethiop. Med. J. 3: 187-190. 
10.,- 1970 . Laboratory and field evaluation of the molluscicidal properties of Phytolacca dodecandra, Bull. WHO 44: 597-612.

11. -, Brody, G., Newel, G. W., Parkhust, R. M., and Skinner, W. A., 1972. Studies on the molluscicidal properties of Endod (Phytolacca dodecandra). 1. Increased potency with butanol extraction, J. Parasitol. 58 (1): 104-107.

12. Medina, F. R. and Ritchie, L. S., Molluscicidal activity of the Puerto Rican weed, Solanum nodiflorum against the snail hosts of Fasciola hepatica. Unpublished MS.

13. Michelson, E. H. and Augustine, D. L., 1957. Studies on the biological control of schistosome-bearing snails. V. The control of Biomphalaria pfeifferi populations by the snail, Marisa cornuarietis, under laboratory conditions, J. Parasitol. 43 (2): 135.

14. Morais, J. G., Almeida, A. M. Barbosa, F. S., and Calado, O. B., 1953. Synergistic molluscicidal action of the saponin from Sapindus saponaria and sodium pentachlorophenate, Ann. 10th Congr. Brasil Hig. Belo Horizonte, 370-373.

15. Palmer, J. R. and Ferguson, F. F., 1965. Present possibilities for bilharzia control in Puerto Rico. Unpublished MS.

16. Peebles, C. R., Oliver-González, J., and Ferguson, F. F., 1972. Apparent adverse effect of Marisa cornuaritis upon Lymnaea columella and Biomphalaria glabrata in an ornamental pond in Puerto Rico, Proc. Hawaiian Ent. Soc. 21 (2): 247-256.

17. Ruíz-Tiben, E., Palmer, J. R., and Ferguson, F. F., 1969. Biological control of Biomphalaria glabrata by Marisa cornuarietis in irrigation ponds of Puerto Rico, Bull. WHO 41: 329-333.

18. Warren, K. S. and Peters, P. A., 1968. Cercariae of Schistosoma mansoni and plants: Attempt to penetrate Phaseolus vulgaris and Hedychium coronarium produces a cercaricide, Nature (London) 217: (5129) 692. 